\title{
Wavelet-based pre-filtering for low cost inertial sensors
}

\begin{abstract}
This study proposed to de-noise the IMU signal by effectively band-limiting the signal at the output of each inertial measurement sensor prior to its mechanization and further processing by the Strapdown INS (SDINS) algorithm. Wavelet Multi-Resolution Algorithm (WMRA) is utilized to improve the performance of the inertial sensors by removing their short term noise. The aim of this study is to reveal how WMRA is utilized to improve the performance of the inertial measurement unit systems and investigate how wavelet analysis can be used to analyse and de-noise output of the low-cost inertial sensors. The proposed multi-level decomposition was applied to real accelerometer and gyroscopes data obtained from MEMS IMU (MotionPak II). Different level of decomposition and thresholding filter was evaluated to obtain optimal results. Analysis of the results demonstrate reducing the INS position and velocity error for the specific IMU.
\end{abstract}

Keyword: Global positioning system; Inertial measurement unit; Strapdown inertial navigation system; Vehicular navigation; Wavelet multi-resolution algorithm 